\title{
NUEVO REGISTRO DE Imantodes chocoensis (SQUAMATA: DIPSADIDAE) EN EL CHOCÓ BIOGEOGRÁFICO COLOMBIANO
}

\author{
NEW RECORD OF Imantodes chocoensis (SQUAMATA: DIPSADIDAE) IN THE \\ BIOGEOGRAPHYC CHOCO COLOMBIAN
}

\author{
ECHAVARRÍA-R. JONARD DAVID, ${ }^{1 *}$ Biol, RENTERÍA-M. LUIS ELADIO, ${ }^{1}$ Biol, RENGIFO-M. JHON \\ TAILOR, ${ }^{1}$ M.SC.
}

${ }^{1}$ Universidad Tecnológica del Chocó, Facultad de Ciencias Básicas y Exactas, Grupo de Investigación en Herpetología, Quibdó, Colombia.

\section{Key words:}

Biodiversity, taxonomy, Neotropics, snakes.

\section{Abstract}

In order to clarify taxonomic States associated with the genus Imantodes in the scientific collection of Zoological reference of the Chocó-herpetology of the technological University of Chocó, it was a review of 36 specimens deposited in it. Identified four species with taxonomic course, I. cenchoa, I. inornatus, I. lentiferus, e I. chocoensis; is last is a new additional record for the Chocó biogeographical Colombian, based on two specimens collected in areas of tropical rain forest (bp-T) corresponding to the corregimiento of Pacurita, municipality of Quibdo and Samurindo, the Atrato municipality. He is required then the distribution of the species, while previous studies mentioned its presence in the region, but without town points out in the Department of Choco (Quibdo). Additionally, some aspects are discussed on their conservation status.

\section{Palabras Clave:}

Biodiversidad, taxonomía, Neotrópico, serpientes.
INFORMACIÓN Recibido: 18-02-2016; Aceptado: 31-03-2016. Correspondencia autor: jd07echavarria@hotmail.com

\section{Resumen}

Con el fin de clarificar estados taxonómicos asociados al género Imantodes en la Colección Científica de Referencia Zoológica del Chocó-Herpetología de la Universidad Tecnológica del Chocó, se efectuó una revisión de 36 ejemplares depositados en ella. Se determinaron cuatro especies con duda taxonómica, I. cenchoa, I. inornatus, I. lentiferus, e $I$. chocoensis; está última constituye un nuevo registro adicional para el Chocó biogeográfico colombiano, con base en dos ejemplares colectados en zonas de bosques pluvial tropical (bp-T) correspondiente a los corregimiento de Pacurita, municipio de Quibdó y Samurindó, municipio del Atrato. Se precisa entonces la distribución de la especie, a pesar de que estudios previos mencionan su presencia en la región pero sin localidad precisa en el departamento del Chocó (Quibdó). Adicionalmente, se discuten algunos aspectos sobre su estado de conservación. 


\section{Introducción}

El género Imantodes, ubicado dentro de la subfamilia Dipsadinae (PYRON et al., 2013; UETZ y HOŠEK, 2015), comprende especies arbóreas, nocturnas y con reproducción ovípara, que se alimentan de anfibios y pequeños lagartos. Estas serpientes comúnmente son conocidas como "serpientes de vid" o serpientes de arbustos, las cuales se distribuyen desde México hasta Argentina (MYERS, 1982).

Pocas serpientes pueden confundirse con las especies del género debido a que presentan una serie de características particulares que hacen fácil distinguirlas de todas las demás serpientes del nuevo mundo. Estas características son: cuerpo lateralmente comprimido, de sección transversal piramidal y cabeza ancha, corta y visible y diferenciable del cuerpo, por su estrecho cuello largo, ojos prominentes, con pupilas elípticas, entre otras. El género incluye actualmente ocho especies (UETZ y HOŠEK, 2015), I. cenchoa (LINNAEUS, 1758), I. gemmistratus (COPE, 1861), I. tenuissimus
(COPE, 1867), I. lentiferus (COPE, 1894), I. inornatus (BOULENGER, 1896), I. phantasma (MYERS, 1982), l. chocoensis (TORRES-CARVAJAL et al., 2012) e $I$. guane (MISSASSI y PRUDENTE, 2015).

\section{Materiales y métodos}

Se examinaron 36 ejemplares del género Imantodes depositados en la Colección Científica de Referencia Zoológica del Chocó-Herpetología de la Universidad Tecnológica del Chocó "Diego Luis Córdoba" (UTCH: COLZOOCH). Los ejemplares fueron colectados en proyectos de investigación y trabajos de grado realizados por investigadores y estudiantes del programa de Biología de la Universidad Tecnológica del Chocó respectivamente. La determinación taxonómica se efectuó en el laboratorio de herpetología de esta misma institución, con base en PETERS y DONOSO-BARROS (1970), TORRES-CARVAJAL et al. (2012), JARAMILLO-MARTÍNEZ et al. (2013) y por comparación con material de referencia de la colección antes mencionada (Tabla 1).

Tabla 1. Ejemplares examinados depositados en la Colección Científica de Referencia Zoológica del Chocó, Herpetología de la Universidad Tecnológica del Chocó.

\begin{tabular}{|c|c|c|c|}
\hline Especies & $\mathrm{N}^{\circ}$ Colección & Coordenadas & Localidad \\
\hline \multirow[t]{2}{*}{ Imantodes chocoensis } & UTCH: COLZOOCH-H: 0275 & $5^{\circ} 40^{\prime} 60^{\prime \prime}, 76^{\circ} 40^{\prime} 0^{\prime \prime}$ & Pacurita (Cabí) \\
\hline & UTCH: COLZOOCH-H: 0318 & $5^{\circ} 35^{\prime} 15^{\prime \prime}, 76^{\circ} 27^{\prime} 6^{\prime \prime}$ & Samurindó \\
\hline \multirow{3}{*}{ Imantodes inornatus } & UTCH: COLZOOCH-H: 0219 & $5^{\circ} 41^{\prime}, 76^{\circ} 40^{\prime}$ & Pacurita (Cabí) \\
\hline & UTCH: COLZOOCH-H: 1499 & $5^{\circ} 21^{\prime} 37.4^{\prime \prime}, 76^{\circ} 38^{\prime} 45.3^{\prime \prime}$ & Salero \\
\hline & UTCH: COLZOOCH-H: 2222 & $5^{\circ} 19^{\prime} 11.22^{\prime \prime}, 76^{\circ} 37^{\prime} 4.27^{\prime \prime}$ & Salero \\
\hline \multirow[t]{2}{*}{ Imantodes lentiferus } & UTCH: COLZOOCH-H: 1413 & \multirow{2}{*}{$5^{\circ} 21^{\prime} 37.4^{\prime \prime}, 76^{\circ} 38^{\prime} 45.3^{\prime \prime}$} & Salero \\
\hline & UTCH: COLZOOCH-H 1429 & & Salero \\
\hline \multirow[t]{29}{*}{ Imantodes cenchoa } & UTCH:COLZOOCH-H:0078 & $5^{\circ} 41^{\prime}, 76^{\circ} 40^{\prime}$ & San Isidro \\
\hline & UTCH:COLZOOCH-H:0263 & $5^{\circ} 37^{\prime} 60^{\prime \prime}, 76^{\circ} 33^{\prime} 0^{\prime \prime}$ & San José de Purré \\
\hline & UTCH:COLZOOCH-H:0293 & $5^{\circ} 40^{\prime} 60^{\prime \prime}, 76^{\circ} 40^{\prime} 0^{\prime \prime}$ & San Martín de Purré \\
\hline & UTCH:COLZOOCH-H:0294 & $5^{\circ} 40^{\prime} 60^{\prime \prime}, 76^{\circ} 40^{\prime} 0^{\prime \prime}$ & San Martín de Purré \\
\hline & UTCH:COLZOOCH-H:0295 & $5^{\circ} 40^{\prime} 60^{\prime \prime}, 76^{\circ} 40^{\prime} 0^{\prime \prime}$ & San Martín de Purré \\
\hline & UTCH:COLZOOCH-H:0317 & $5^{\circ} 35^{\prime} 15^{\prime \prime}, 76^{\circ} 27^{\prime} 6^{\prime \prime}$ & Samurindó \\
\hline & UTCH:COLZOOCH-H:0583 & $8^{\circ} 13^{\prime}, 77^{\circ} 20^{\prime}$ & Cerro Tacarcuna \\
\hline & UTCH:COLZOOCH-H: 1168 & & San Roque \\
\hline & UTCH:COLZOOCH-H: 1169 & & Nauca \\
\hline & UTCH:COLZOOCH-H: 1181 & $5^{\circ} 19^{\prime} 36.1^{\prime \prime}, 76^{\circ} 26^{\prime} 41.3^{\prime \prime}$ & Angostura \\
\hline & UTCH:COLZOOCH-H: 1416 & $5^{\circ} 21^{\prime} 37.4^{\prime \prime}, 76^{\circ} 38^{\prime} 45.3^{\prime \prime}$ & Salero \\
\hline & UTCH:COLZOOCH-H: 1420 & $5^{\circ} 21^{\prime} 37.4^{\prime \prime}, 76^{\circ} 38^{\prime} 45.3^{\prime \prime}$ & Salero \\
\hline & UTCH:COLZOOCH-H: 1423 & $5^{\circ} 21^{\prime} 37.4^{\prime \prime}, 76^{\circ} 38^{\prime} 45.3^{\prime \prime}$ & Salero \\
\hline & UTCH:COLZOOCH-H: 1431 & $5^{\circ} 21^{\prime} 37.4^{\prime \prime}, 76^{\circ} 38^{\prime} 45.3^{\prime \prime}$ & Salero \\
\hline & UTCH:COLZOOCH-H: 1445 & $5^{\circ} 21^{\prime} 37.4^{\prime \prime}, 76^{\circ} 38^{\prime} 45.3^{\prime \prime}$ & Salero \\
\hline & UTCH:COLZOOCH-H: 1478 & $5^{\circ} 21^{\prime} 37.4^{\prime \prime}, 76^{\circ} 38^{\prime} 45.3^{\prime \prime}$ & Salero \\
\hline & UTCH:COLZOOCH-H: 1559 & $5^{\circ} 41^{\prime} 11^{\prime \prime}, 76^{\circ} 38^{\prime} 07^{\prime \prime}$ & Pacurita (Cabí) \\
\hline & UTCH:COLZOOCH-H: 1560 & $5^{\circ} 41^{\prime} 11^{\prime \prime}, 76^{\circ} 38^{\prime} 07^{\prime \prime}$ & Pacurita (Cabí) \\
\hline & UTCH:COLZOOCH-H: 1646 & $5^{\circ} 41^{\prime} 11^{\prime \prime}, 76^{\circ} 38^{\prime} 07^{\prime \prime}$ & Pacurita (Cabí) \\
\hline & UTCH:COLZOOCH-H: 1647 & $5^{\circ} 41^{\prime} 11^{\prime \prime}, 76^{\circ} 38^{\prime} 07^{\prime \prime}$ & Pacurita (Cabí) \\
\hline & UTCH:COLZOOCH-H: 1648 & $5^{\circ} 41^{\prime} 11^{\prime \prime}, 76^{\circ} 38^{\prime} 07^{\prime \prime}$ & Pacurita (Cabí) \\
\hline & UTCH:COLZOOCH-H: 1649 & $5^{\circ} 41^{\prime} 11^{\prime \prime}, 76^{\circ} 38^{\prime} 07^{\prime \prime}$ & Pacurita (Cabí) \\
\hline & UTCH:COLZOOCH-H: 1650 & $5^{\circ} 41^{\prime} 11^{\prime \prime}, 76^{\circ} 38^{\prime} 07^{\prime \prime}$ & Pacurita (Cabí) \\
\hline & UTCH:COLZOOCH-H: 1651 & $5^{\circ} 41^{\prime} 11^{\prime \prime}, 76^{\circ} 38^{\prime} 07^{\prime \prime}$ & Pacurita (Cabí) \\
\hline & UTCH:COLZOOCH-H: 2098 & $5^{\circ} 42^{\prime} 17.3^{\prime \prime}, 76^{\circ} 26^{\prime} 25.8^{\prime \prime}$ & Salero \\
\hline & UTCH:COLZOOCH-H: 2128 & $5^{\circ} 42^{\prime} 17.3^{\prime \prime}, 76^{\circ} 26^{\prime} 25.8^{\prime \prime}$ & Salero \\
\hline & UTCH:COLZOOCH-H: 2217 & $5^{\circ} 19^{\prime} 11.22^{\prime \prime}, 76^{\circ} 37^{\prime} 4.27^{\prime \prime}$ & \\
\hline & UTCH:COLZOOCH-H: 2245 & $8^{\circ} 1^{\prime} 30^{\prime \prime}, 77^{\circ} 9^{\prime} 25^{\prime \prime}$ & Unguía \\
\hline & UTCH:COLZOOCH-H: 2246 & $8^{\circ} 1$ '30", 77ㅇ' $25^{\prime \prime}$ & Unguía \\
\hline
\end{tabular}


Adicionalmente, se revisaron bases de datos de las siguientes colecciones colombianas: Instituto de Investigación de Recursos Biológicos Alexander von Humboldt (IAvH), Museo de Herpetología de la Universidad de Antioquia (MHUA), Colección de Reptiles del Instituto de Ciencias Naturales de la Universidad Nacional de Colombia (ICN), Universidad del Valle (UVC), de donde se obtuvieron registros de presencia de la especie en territorio colombiano, que junto a nuestros registros fueron utilizados para construir un mapa de puntos de presencia comprobada para la especie.

\section{Resultados y discusión}

Producto de la revisión del material se determinaron cuatro especies del género Imantodes para el departamento del Chocó: I. cenchoa, I. inornatus, I. lentiferus e I. chocoensis, está última objeto de estudio en esta investigación (Fig. 1), basada en dos especímenes identificados con número de colección UTCH: COLZOOCH-H 0275, UTCH: COLZOOCH 0318, que habían sido previamente asignados a I. cenchoa e I. inornatus respectivamente.

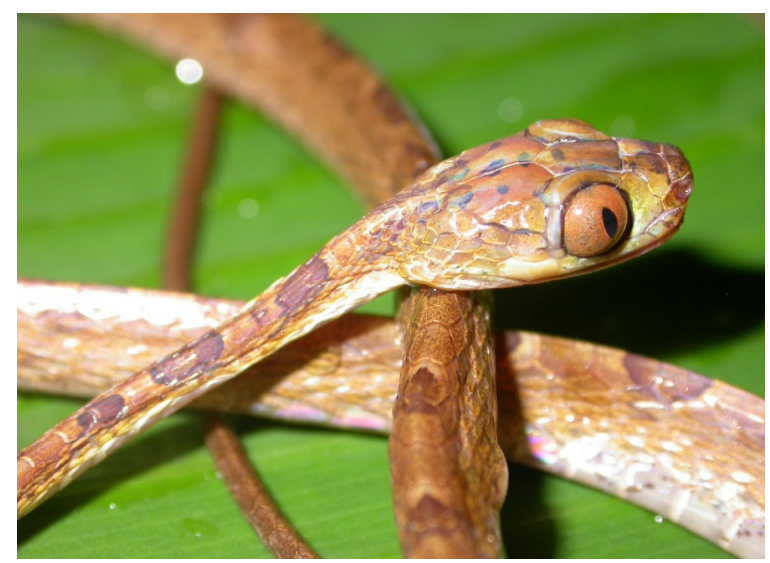

Figura 1. Espécimen en vida de I. chocoensis (UTCH: COLZOOCH-H 0275), colectado en el corregimiento de Pacurita, municipio de Quibdó. Chocó-Colombia (540'31.40" N, 76³7'15.09" W, 53 m). Foto: Alex Mauricio Jiménez-Ortega.

El primer ejemplar aquí registrado corresponde a un macho adulto, colectado en el corregimiento de Pacurita municipio de Quibdó (540'31.40'”N, 76³7'15'”, $53 \mathrm{~m}$ ), el 10 de junio de 2003 por Franklin De Jesús Murillo-Moreno (UTCH: COLZOOCH-H 275). El segundo ejemplar hace referencia a una hembra adulta colectada en el corregimiento de Samurindó municipio del Atrato (534'57" N, 76³9'55” W, 30 m), en junio de 2009 por Jonard David Echavarría-Rentería y Jorge
Iván Delgado Franco (UTCH: COLZOOCH-H 318), el material colectado se encuentra en buen estado de conservación (Fig. 2).

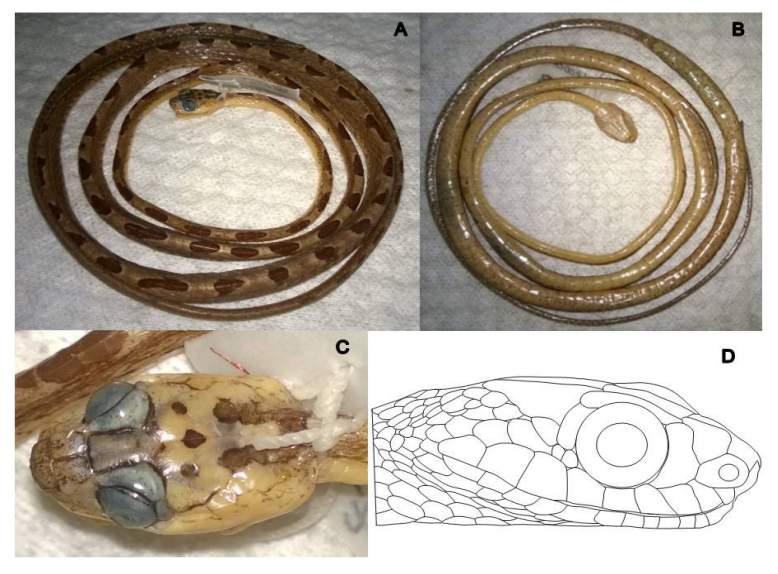

Figura. 2. (A) Vista dorsal, (B) vista ventral, (C) vista dorsal de la cabeza y (D) llustración de patrones de escamación en la cabeza (Ausencia de escama loreal) de I. chocoensis colectado en zona de depositado en colección, registrado, departamento del Chocó-Colombia.

Ambas localidades están ubicadas en La zona central del departamento del Chocó, que corresponde a la zona de vida de bosques pluvial tropical (bp-T), caracterizada por presentar una precipitación anual de $8000-10000 \mathrm{~mm}$, temperatura megatermal que oscila entre $26^{\circ} \mathrm{C}$ a $28^{\circ} \mathrm{C}$ y una húmeda relativa de $91.8 \%$ (HOLDRIDGE, 1996, RANGEL-CH., 2004, BOLAÑOS et al. 2005). Los sitios de colecta de los ejemplares (UTCH: COLZOOCH-H 275, UTCH: COLZOOCH-H 318), actualmente enfrentan procesos de degradación debido a la explotación de oro y platino a través de actividades como la minería a cielo abierto y tala de madera, actividades que junto a la cacería y el sistema de "tumbe y pudre" de pequeñas parcelas para cultivos, han transformando el bosque original en diversos paisajes o mosaicos, lo que se convierte en un factor que afecta a esta especie y pone en riesgo la viabilidad de sus poblaciones (Problemática que se extiende para la mayoría de las serpientes presentes en el departamento del Chocó).

I. chocoensis es una especie descrita para el noroeste de Ecuador en la localidad de Durango Provincia Esmeraldas (0101'41.880" N, 78³5'42.000" W, 253 msnm) por TORRES-CARVAJAL et al. (2012), trabajo que ha sido fundamental para esclarecer los problemas taxonómicos asociados a este género particularmente en Colombia, ya que hasta años recientes sólo se hablaba de tres especies con presencia en el país y gran parte de los especímenes depositados en las colecciones correspondientes a esta especie 
que habían sido determinados como $I$. inornatus e Imantodes sp., debido a que en su momento no se hizo énfasis en caracteres importantes como la escama loreal y el patrón de manchas dorsales que presenta cada especie, además de otras características que presentan.

De allí que los registros conocidos a la fecha para Colombia han sido sumados por JARAMILLOMARTÍNEZ et al. (2013) quienes documentan la presencia de I. chocoensis para la Quebrada El Caimancito, corregimiento de Silva y San Vicente (UV-C-7249) y en el Corregimiento de PianguitaBazán del municipio de Buenaventura, departamento del Valle del Cauca. Este último basado en registros fotográficos obtenidos en septiembre de 2011. Así mismo ROJAS-MORALES et al. (2013) mencionaban un registro de un individuo para la Reserva Forestal San Cipriano-Escalerete del municipio de Buenaventura, departamento del Valle del Cauca (IAvH-R 6005), el 20 de diciembre de 2008. MISSASSI et al. (2015); MISSASSI y PRUDENTE (2015) registran tres individuos de la especie para los departamentos del Valle del Cauca (ICN 434), colectado el 9 de marzo de 1960), Antioquia (ICN, 1830), registrado el 1 de febrero de 1981 en Amparradó y Chocó (ICN, 120) registrada en octubre de 1947 sin localidad precisa en el municipio de Quibdó (Tabla 2, Fig. 3).

Los registros constituyeron una adición importante en la extensión ecológica de la especie, no obstante, aquí aportamos dos nuevas localidades para $I$. chocoensis en el Chocó biogeográfico colombiano más precisamente en los corregimientos de Pacurita del municipio de Quibdó y Samurindó del municipio del Atrato en el departamento del Chocó, Colombia.

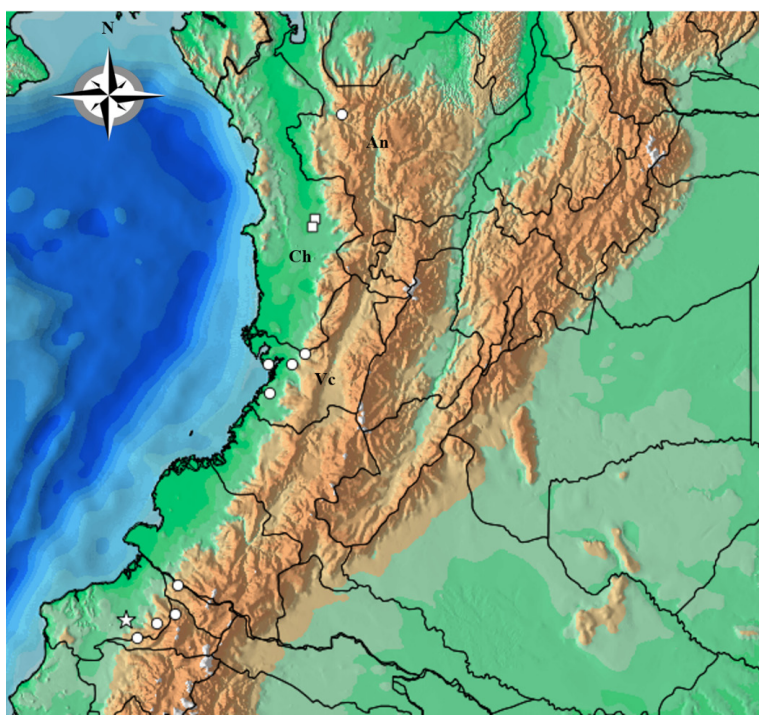

Figura 3. Localización geográfica de los registros de $I$. chocoensis en Ecuador y Colombia: Valle del Cauca (Vc), Antioquia (An) y Chocó (Ch). Estrella blanca: localidad tipo (Torres-Carvajal et al. 2012) en Ecuador. Círculos blancos: Registros de literatura (Jaramillo-Martínez et al. 2013, Rojas-Morales et al. 2013, Missassi, Prudente, 2015 y Missassi et al. 2015). Cuadros blancos: Nuevo registro para el Chocó Biogeografico colombiano. Mapa obtenido de QGIS (Sistema de Información Geográfica para el Ordenamiento Territorial, Colombia).

El hallazgo de esta especie en el Chocó, representa un hecho importante en términos de conservación de los bosques para la preservación de las serpientes de las tierras bajas del Pacífico colombiano. Además, es importante resaltar la existencia de $I$. chocoensis en el país era predecible como sugirió Torres-Carvajal et al. (2012), debido a la continuidad del bosque tropical Chocoano entre el suroeste de Colombia y el noroeste

Tabla 2. Localidades donde se han realzado registros del ejemplar de I. chocoensis en Colombia.

\begin{tabular}{|c|c|c|c|c|c|c|}
\hline Especie & Sexo & $\mathrm{N}^{\circ}$ colección & Localidad & Coordenadas & Altitud & Referencias \\
\hline & M & UTCH: COLZOOCH-H 275 & Pacurita & $5^{\circ} 40^{\prime} 31.40^{\prime \prime} \mathrm{N}, 76^{\circ} 37^{\prime} 15^{\prime \prime} \mathrm{W}$ & $53 \mathrm{~m}$ & \\
\hline $\begin{array}{l}\text { Imantodes } \\
\text { chocoensis }\end{array}$ & $\mathrm{H}$ & UTCH: COLZOOCH-H 318 & Samurindó & $5^{\circ} 34^{\prime} 57^{\prime \prime} \mathrm{N}, 76^{\circ} 39^{\prime} 55^{\prime \prime} \mathrm{W}$ & $30 \mathrm{~m}$ & Este trabajo \\
\hline
\end{tabular}

\begin{tabular}{|c|c|c|c|c|c|}
\hline \multicolumn{6}{|c|}{ Distribución en Colombia } \\
\hline Departamento & Municipio & Localidad & Coordenadas & Altura & Referencias \\
\hline \multirow{3}{*}{ Valle del Cauca } & & Quebrada El Caimancito & $3^{\circ} 28^{\prime} 24.85^{\prime \prime} \mathrm{N}, 77^{\circ} 11^{\prime} 9,82^{\prime \prime} \mathrm{W}$ & $75 \mathrm{~m}$ & \\
\hline & Buenaventura & Pianguita-Bazán & $3^{\circ} 50^{\prime} 32.55^{\prime \prime} \mathrm{N}, 77^{\circ} 11^{\prime} 54.60^{\prime \prime} \mathrm{W}$ & $25 \mathrm{~m}$ & JARAMILLO-MARTÍNEZ et al. 2013 \\
\hline & & San Cipriano-Escalerete Reserva Forestal & $3^{\circ} 50^{\prime} 3.83^{\prime \prime} \mathrm{N}, 76^{\circ} 53^{\prime} 18^{\prime \prime} \mathrm{W}$ & $40 \mathrm{~m}$ & ROJAS-MORALES et al. 2013 \\
\hline Chocó & Quibdó & Quibdó & $5^{\circ} 41^{\prime} 44.28^{\prime \prime} \mathrm{N}, 76^{\circ} 38^{\prime} 59.32^{\prime \prime} \mathrm{W}$ & $43 \mathrm{~m}$ & MISSASSI y PRUDENTE, 2015 \\
\hline Valle del Cauca & Darién & Río Calima & $3^{\circ} 59^{\prime} 12.99^{\prime \prime} \mathrm{N}, 76^{\circ} 46^{\prime} 25.69^{\prime \prime} \mathrm{W}$ & $396 \mathrm{~m}$ & \\
\hline Antioquía & Dabeiba & Amparradó & $\begin{array}{c}7^{\circ} 0^{\prime} 8.09^{\prime \prime} \mathrm{N}, \\
76^{\circ} 15^{\prime} 54.577^{\prime \prime} \mathrm{W}\end{array}$ & $763 \mathrm{~m}$ & MISSASSI et al. 2015 \\
\hline
\end{tabular}


de Ecuador. De las cinco especies de Imantodes registradas para Colombia, en esta investigación se determinaron cuatro, las mismas reportadas para las selvas de las tierras bajas del Pacífico, especies que son probablemente simpátricas en estas localidades en el departamento del Chocó.

Referente al estado de conservación de I. chocoensis, a la fecha no se encuentran en ningún Apéndice CITES (2014), no obstante, según la UICN (2015) se encuentra bajo la categoría de preocupación menor (LC). Debido a los vacíos de información que existen sobre aspectos de su ecología, biología, taxonomía de las especies del género y sus relaciones filogenéticas, se sugiere reevaluar su categorización. En este sentido, sugerimos fortalecer a profesionales o a fines en la taxonomía de estas especies y otras especies de serpientes en el País.

Agradecimientos: A la comunidad de Pacurita y Samurindó que permitieron realizar estas investigaciones. A la Universidad Tecnológica del Chocó "Diego Luis Córdoba", Quibdó, por ser una vez más una institución que apoya las investigaciones científicas en el departamento del Chocó, además por todo lo enseñado durante los estudio de pregrado, a Leison Palacios-Mosquera del Grupo de Investigación en Manejo de Fauna Silvestre Chocoana (FASCHO), por su contribución a la mejora del manuscrito, y por último a el Grupo de Investigación en Herpetología de la Universidad Tecnológica del Chocó por su apoyo incondicional.

\section{Referencias}

BOLAÑOS, R.; WATSON, V., TOSI, J. 2005. Mapa ecológico de Costa Rica (Zonas de Vida), según el sistema de clasificación de zonas de vida del mundo de L.R. Holdridge), Escala 1:750 000. Centro Científico Tropical, San José, Costa Rica.

BOULENGER, G.A. 1896. Catalogue of the snakes in the British Museum, Vol. 3. London (Taylor \& Francis), XIV +727 pp. England.

CASTAÑO, O.; CÁRDENAS, G.; HERNÁNDEZ, E.; CASTRO, F. 2004. Reptiles en el Chocó biogeográfico. Págs. 599- 632. En: Rangel, J.O. (Ed.). Diversidad Biótica IV. El chocó Biogeográfico/Costa Pacífica. Universidad Nacional de Colombia, Instituto de Ciencias Naturales, Conservación Internacional. Bogotá, Colombia.

COPE, E.D. 1861. Contributions to the ophiology of Lower California, Mexico and Central America. Proc. Acad. Nat. Sci. Philadelphia 13:292-306.

COPE, E.D. 1867. Fifth contribution lo the herpetology of tropical America. Proc. Acad. nat. Sci. Philadelphia 181866:317-323.

COPE, E.D. 1894. On the species of Himantodes Dumeril and Bibron. American Naturalist 28:612-614. CITES-CONVENCIÓN SOBRE COMERCIO INTERNACIONAL DE ESPECIES SILVESTRES DE FAUNA Y FLORA. Lista de Especies CITES. Diposnibl en: www.cites.org/esp/app/2014/S-Appendices-2014-06-12.pdf. Acceso: 13$\underline{08-2015 .}$

HOLDRIDGE, L. 1996. Ecología Basada en Zonas de Vida. Instituto Interamericano de Cooperación para la Agricultura, IICA. San José de Costa Rica.

IUCN, 2015. The IUCN Red List of Threatened Species. Version 2015-4. Disponible en: http://www.iucnredlist.org. Acceso: 19-11-2015.

JARAMILLO-MARTÍNEZ, A.F.; VALENCIA-ZULETA, A.; CASTRO-HERRERA, F. 2013. Imantodes chocoensis Torres-Carvajal, Yánez-Muñoz, Quirola, Smith, and Almendáriz, 2012 (Squamata: Dipsadidae): First records from Colombia. CheckList 9 (5):1070-1071.

LINNAEUS, C. 1758. Systemanaturæ per regnatrianaturæ, secundumclasses, ordines, genera, species, cum characteribus, differentiis, synonymis, locis. Tomus I. Editiodecima, reformata. LaurentiiSalvii, Holmiæ. 10th Edition. England. 
MISSASSI, F.R.; PRUDENTE, L.C. 2015. A new species of Imantodes Duméril, 1853 (Serpentes, Dipsadidae) from the Eastern Cordillera of Colombia Zootaxa 3980 (4):562-574.

MISSASSI, F.R.; COSTA, C.L.; PRUDENTE, L.C. 2015. Range extension of the Chocoan blunt-headed vine snake: Imantodes chocoensis (Serpentes: Dipsadidae) in northwestern Colombia. Salamandra 51 (3):269-272.

MYERS, C.W. 1982. Blunt-headed vine snakes (Imantodes) in Panama, including a new species and other revisionary notes. American Museum Novitates 2738:1-50.

PETERS, J.A.; DONOSO-BARROS R. 1970. Catalogue of the Neotropical Squamata. Part II. Lizards and amphisbaenians. Bulletin of the United States National Museum 297 (2): 1-293.

PYRON, R.A.; BURBRINK, F.T.; WIENS, J.J. 2013. A phylogeny and revised classification of Squamata, including 4161 species of lizards and snakes. BMC Evolutionary Biology, 13, 93. http://dx.doi.org/10.1186/1471-2148-13-93.

RANGEL-CH., J.O. 2004. Colombia Diversidad Biótica, IV, El Chocó Biogeográfico/Costa Pacífica. Instituto de Ciencias Naturales de la Universidad Nacional de Colombia, Conservación Internacional. Bogotá.

ROJAS-MORALES, J.A.; ESCOBAR-LASSO, S.; OSORIO-ORTÍZ, A.; LOZANO-RÍOS, L.A. 2013. Third observation of the Chocoan blunt-headed vine snake, Imantodes chocoensis (Serpentes: Dipsadidae) for Colombia. Biota Colombiana 14:341-344.

TORRES-CARVAJAL, O.; YÁNEZ-MUÑOZ, M.H.; QUIROLA, D.; SMITH, E.N.; ALMENDÁRIZ, A. 2012. A new species of blunt-headed vine snake (Colubridae, Imantodes) from the Choco region of Ecuador. ZooKeys 244:91-110.

UETZ, P.; HOŠEK J. 2015. The Reptile Database. Disponible en: http://www.reptile-database.org. Acceso: 13-08-2015. 